\title{
Drivers of year-to-year variation in exacerbation frequency of COPD: analysis of the AERIS cohort
}

\author{
Tom M.A. Wilkinson ${ }^{1,2,3}$, Emmanuel Aris ${ }^{4}$, Simon C. Bourne ${ }^{1,5}$, Stuart C. Clarke ${ }^{1,3}$, \\ Mathieu Peeters ${ }^{4}$, Thierry G. Pascal ${ }^{4}$, Laura Taddei ${ }^{6}$, Andrew C. Tuck ${ }^{7}$, \\ Viktoriya L. Kim ${ }^{1,2}$, Kristoffer K. Ostridge (10) ${ }^{1,2}$, Karl J. Staples (101,2,3, \\ Nicholas P. Williams ${ }^{1,2}$, Anthony P. Williams ${ }^{3}$, Stephen A. Wootton ${ }^{8}$ and \\ Jeanne-Marie Devaster $\mathbb{1}^{4}$ on behalf of the AERIS Study Group ${ }^{9}$
}

\begin{abstract}
Affiliations: ${ }^{1}$ Clinical and Experimental Sciences, University of Southampton Faculty of Medicine Southampton General Hospital, Southampton, UK. ${ }^{2}$ Southampton NIHR Respiratory Biomedical Research Unit, Southampton General Hospital, Southampton, UK. ${ }^{3}$ Wessex Investigational Sciences Hub, University of Southampton Faculty of Medicine, Southampton General Hospital, Southampton, UK. ${ }^{4}$ GSK, Wavre, Belgium. ${ }^{5}$ Portsmouth Hospitals NHS Trust, Queen Alexandra Hospital, Portsmouth, UK. ${ }^{6}$ GSK, Sienna, Italy. ${ }^{7}$ Faculty of Medicine and Institute for Life Sciences, University of Southampton, Southampton, UK. ${ }^{8}$ Southampton NIHR Biomedical Research Centre, University Hospital Southampton NHS Foundation Trust, Southampton, UK. ${ }^{9}$ Members of the AERIS Study Group are listed in the Acknowledgements section.
\end{abstract}

Correspondence: Jeanne-Marie Devaster, GSK Vaccines, Rue de l'Institut 89, 1330 Rixensart, Belgium. E-mail: jeanne-marie.devasterlagsk.com.

ABSTRACT The association between exacerbation aetiology and exacerbation frequency is poorly understood.

We analysed 2-year follow-up data from a prospective observational study of patients with chronic obstructive pulmonary disease (COPD) (www.clinicaltrials.gov identifier number NCT01360398) to evaluate year-to-year variation in exacerbation frequency and related aetiology. A total of 127 patients underwent blood and sputum sampling monthly and at exacerbation to detect respiratory infections and eosinophilic inflammation; 103 continued into year 2 and 88 completed both years.

The most common bacterial species at stable state and exacerbation was Haemophilus influenzae. Among infrequent exacerbators (one exacerbation per year), the incidence of viral infection at exacerbation was high $(60.0 \%$ (95\% CI 35.1-81.7\%) in year 1 and $78.6 \%(53.4-94.2 \%)$ in year 2). Those with more frequent exacerbations tended to have higher relative incidence of bacterial than viral infection. Patients with at least two additional exacerbations in year 2 versus year 1 had a higher risk of $H$. influenzae colonisation at stable state than those with at least two fewer exacerbations, as detected by culture (OR 1.43 (95\% CI 0.71-2.91) versus 0.63 (0.40-1.01), $\mathrm{p}=0.06$ ) and PCR (1.76 (95\% CI $0.88-3.51$ ) versus 0.56 $(0.37-0.86), \mathrm{p}<0.01)$. This was not seen with other infection types or eosinophilic inflammation.

Analysis of the same cohort over 2 years showed, for the first time, that changes in yearly COPD exacerbation rate may be associated with variations in $H$. influenzae colonisation.

@ERSpublications

Analysis of the same cohort of patients over 2 years showed that changes in yearly COPD exacerbation rate may be associated with variations in Haemophilus influenzae colonisation http://ow.ly/Qe9430nkT7U

Cite this article as: Wilkinson TMA, Aris E, Bourne SC, et al. Drivers of year-to-year variation in exacerbation frequency of COPD: analysis of the AERIS cohort. ERJ Open Res 2019; 5: 00248-2018 [https://doi.org/10.1183/23120541.00248-2018].

This article has supplementary material available from openres.ersjournals.com.

This study is registered at www.clinicaltrials.gov with identifier number NCT01360398. Anonymised individual participant data and study documents can be requested for further research from www.clinicalstudydatarequest.com.

Received: Dec 182018 | Accepted: Dec 192018

Copyright $\odot$ ERS 2019. This article is open access and distributed under the terms of the Creative Commons Attribution Non-Commercial Licence 4.0. 


\section{Introduction}

Acute exacerbations of chronic obstructive pulmonary disease (AECOPD) lead to a faster decline in lung function, worsened quality of life and increased risk of hospitalisation [1] and are a significant prognostic factor of reduced survival across all chronic obstructive pulmonary disease (COPD) stages [2].

Exacerbations may be triggered by respiratory bacterial or viral infections and environmental factors, such as pollution $[1,3]$. Their frequency can be predicted by patients' exacerbation history [4] and is linked to disease severity, with frequent exacerbators experiencing a greater decline in forced expiratory volume in $1 \mathrm{~s}$ over time than infrequent exacerbators [5].

Recent studies have reported an association between exacerbation risk and a shift in the microbiome towards enrichment of Proteobacteria [6-10]. However, little is known about the association between exacerbation aetiology and frequent/infrequent exacerbator phenotypes or the factors that promote a change in exacerbation frequency from one year to the next beyond the effects of prescribed therapies for AECOPD, such as bronchodilator and corticosteroid treatments or macrolide antibiotics [3].

We previously reported first-year results from the prospective longitudinal Acute Exacerbation and Respiratory Infections in COPD (AERIS) study in which COPD patients underwent sputum sampling each month and at exacerbation over 2 years to assess how changes in the airway microbiome contribute to the incidence and severity of AECOPD [9, 11]. During the first year of follow-up, exacerbations were associated with infections with Moraxella catarrhalis and nontypeable Haemophilus influenzae, and with respiratory viruses, particularly human rhinovirus (HRV) [9]. The composition of the lung microbiome showed unique profiles within subtypes of COPD exacerbations, with nonrandom probabilities of repeating particular subtypes [10].

We now present 2-year follow-up data from the AERIS study with the aim to evaluate year-to-year variation in exacerbation frequency and the aetiology related to these events.

A "focus on the patient" section (figure 1) summarises the clinical relevance and impact of this study on the patient population.

\section{Methods}

Study design and procedures

The 2-year prospective, observational cohort AERIS study (www.clinicaltrials.gov registration NCT01360398) was based at University Hospital Southampton, UK. The study protocol has been published [11] and a summary is available at www.gsk-clinicalstudyregister.com (study identifier 114378). AERIS was conducted in accordance with the Declaration of Helsinki and Good Clinical Practice, and was approved by the Southampton and South West Hampshire Research Ethics Committee. All participants provided written informed consent. Results have been reported for subjects followed over 1 year for the primary objective

\section{What is the context?}

Patients with chronic obstructive pulmonary disease (COPD) are prone to acute respiratory exacerbations. During these acute exacerbations of COPD (AECOPD), patients experience worsening symptoms, most commonly shortness of breath, cough and airflow obstruction. Bacterial and viral infections are important triggers of AECOPD.

\section{What is new?}

We studied a group of patients with COPD over a period of 2 years, taking sputum samples monthly and at exacerbation to detect bacteria and viruses. Results from the first year of study showed exacerbations were mainly associated with infections with Moraxella catarrhalis and Haemophilus influenzae bacterial species, and respiratory viruses, particularly human rhinovirus. Results from the second year confirm these findings. Also, by comparing year 1 and year 2 results for the same group of patients over 2 years, we showed for the first time that a change in yearly exacerbation rate may be associated with variations in $\mathrm{H}$. influenzae colonisation.

\section{What is the impact?}

We confirmed the importance of $H$. influenzae as a cause of exacerbations in patients with COPD, suggesting this bacterium should be targeted to reduce the risk of AECOPD.

FIGURE 1 Focus on the patient. 
(incidences of AECOPD and AECOPD with sputum containing bacterial pathogens), and on incidences of bacterial and viral pathogens and eosinophilic inflammation in AECOPD and stable state $[9,10,12]$.

Study procedures and definitions of AECOPD and COPD severity categories were described previously [9]. Briefly, patients aged 40-85 years with a confirmed diagnosis of COPD, categorised as moderate, severe or very severe $[11,13]$, were recruited from June 2011 to June 2012. Patients were followed monthly for 24 months in the stable state and reviewed within $72 \mathrm{~h}$ of onset of AECOPD symptoms. Exacerbations were detected using daily electronic diary cards.

Sputum samples were obtained by spontaneous expectoration or induced and were processed according to standard methods, as described previously [9]. COPD exacerbation subtypes were classified as bacterial, viral or eosinophilic, using specific criteria [14]: bacterial, if at least one positive potentially pathogenic bacteria culture was isolated (H. influenzae, M. catarrhalis, Streptococcus pneumoniae, Pseudomonas aeruginosa or Staphylococcus aureus); viral, if at least one positive viral PCR result; eosinophilic, if eosinophils were $>3 \%$ of nonsquamous cells from sputum. Bacterial colonisation was indicated by bacterial presence at stable state.

\section{Statistical analysis}

The sample size calculation was described previously [11]. Results are presented for the full cohort, defined as all patients considered by the investigator as eligible for study procedures and excluding those who withdrew consent at the first visit. In descriptive analyses that compared follow-up results from the first year of the study to those from the second year (months 13 to 24), patients who completed both years of the study (completer cohort) were considered as well as data for the full cohort.

The percentage of stable-state and exacerbation-state sputum samples containing bacterial or viral pathogens or eosinophils $>3 \%$ was calculated with $95 \%$ confidence intervals computed using the generalised linear model assuming a negative binomial distribution for the response variable with logarithm as link function, and the logarithm of time for follow-up as an offset variable.

To examine the relationship between the number of exacerbations each year and the presence of bacteria, viruses or eosinophils $>3 \%$, generalised estimating equation regression models with random intercept were used. These analyses should be considered post hoc as they were not included in the AERIS statistical analysis plan.

Statistical analysis was performed using the SAS Drug Development platform version 4.3.2 (SAS Institute, Cary, NC, USA).

\section{Results}

Study population

Of the initial 127 subjects, 103 entered year 2 (full cohort, year 2) and 88 completed all follow-up visits (completer cohort) (figure 2). There were no major differences in baseline characteristics, including COPD severity, among the study cohorts (table 1). Rates of sputum collection in year 1 and year 2 were high (figure 2). In both years, most AECOPD samples (67\% in year 2 and $71 \%$ in year 1 ) were collected within 2 days of the start of exacerbation symptoms.

Most sputum samples were taken before antibiotic administration. In year 2, no antibiotic had been administered before sputum collection for $91.3 \%$ (619 out of 678 ) stable and $88.7 \%$ (173 out of 195) exacerbation samples and, in year 1, for 98.9\% (948 out of 959) stable and $91.4 \%$ (296 out of 324) exacerbation samples. The percentage of follow-up time during which patients received azithromycin was $3.0 \%$ in year 1 and $11.5 \%$ in year 2 at stable state. Eosinophilic inflammation (eosinophils $>3 \%$ ) was present in $35.5 \%$ of sputum samples at stable state and $24.7 \%$ at exacerbation in year 1 , and $33.1 \%$ and $24.3 \%$, respectively, in year 2 .

\section{Incidence and aetiology of AECOPD}

In year 1 , the mean exacerbation rate was 3.04 (95\% CI $2.63-3.50)$ per patient-year and $2.32(95 \% \mathrm{CI}$ 1.99-2.72) per patient-year in the 103 patients who continued into year 2. A total of 223 acute exacerbations were recorded in year 2 and $60.2 \%$ of patients had two or more exacerbations (figure 3 and table S1). During the first year of follow-up, 355 acute exacerbations were recorded and $61.4 \%$ of patients had two or more exacerbations. The number of exacerbations missed during year 1 and year 2 was 27 and 29 , respectively. In both years, most exacerbations were moderate in severity (191 (85.6\%) in year 2 versus $304(85.6 \%)$ in year 1). Among the 33 patients with five or more exacerbations in year 1 (figure 3), seven (21\%) discontinued the study. Among the 88 completers, in year 2 versus year 1, 32 (36\%) had at least two fewer exacerbations, 13 (15\%) had one fewer, 15 (17\%) had no change, $13(15 \%)$ had one more and 15 (17\%) had at least two more events. 


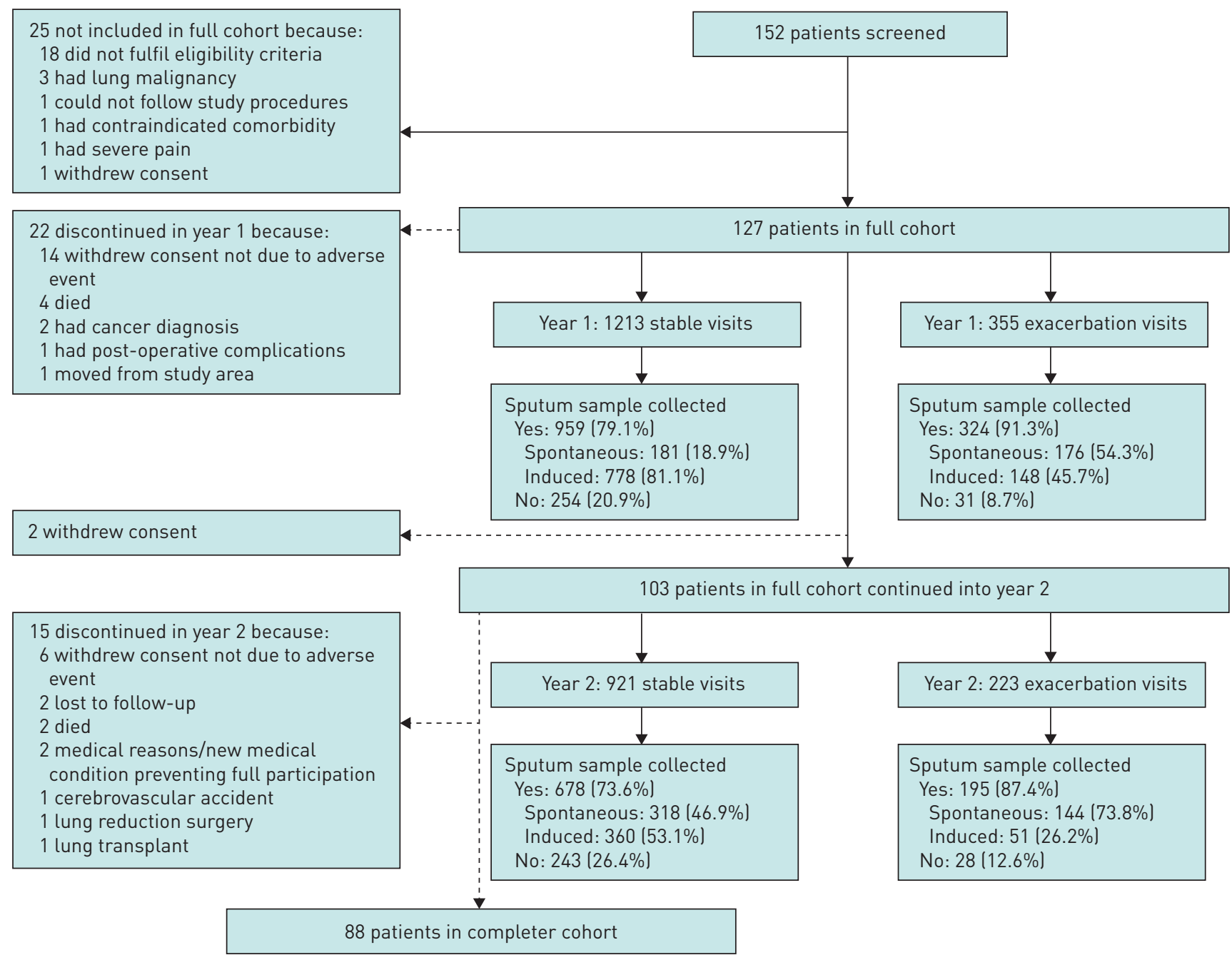

FIGURE 2 Flow chart of patients and sputum sampling in the study.

The most common bacterial species detected by sputum culture and/or PCR in both years was $H$. influenzae (figure 4 and table S2). As in year 1, the percentage of samples in year 2 that were positive by culture for $H$. influenzae or $M$. catarrhalis was higher at exacerbation than at stable state (figure 4). With PCR detection, no increase was found in year 2 in the percentage of samples positive for H. influenzae (41.0\% (95\% CI 36.9-45.2\%) at stable state versus 39.0\% (31.8-46.6\%) at exacerbation), while an increase was observed for $M$. catarrhalis $(12.5 \%$ (95\% CI 9.9-15.6\%) at stable state versus $17.5 \%$ (95\% CI 12.2-23.9) at exacerbation).

As in year 1, the percentage of sputum samples positive for at least one virus increased from stable state to exacerbation in year 2 (from $11.0 \%$ to $33.3 \%$ ) (figure 4). As expected, the incidence of specific viral pathogens varied from year to year but the most common species detected was HRV, in $6.4 \%$ and $18.1 \%$ of stable and exacerbation samples, respectively.

\section{Aetiology of AECOPD relative to exacerbator frequency}

We examined the presence of bacteria relative to the presence of viral pathogens or eosinophilic sputum samples and number of exacerbations reported each year per patient in the completer cohort. In year 1, among patients with collected sputum, 17 were considered infrequent exacerbators (one exacerbation), 30 were frequent exacerbators (two to four exacerbations) and 24 were very frequent exacerbators (more than four exacerbations). In year 2, 16 patients were infrequent exacerbators, 42 were frequent exacerbators and nine were very frequent exacerbators.

At exacerbation, infrequent exacerbators tended to have a lower incidence of bacterial infection relative to viral infection, while those with more frequent exacerbations tended to have a higher incidence of bacterial 


\begin{tabular}{|c|c|c|c|}
\hline Characteristic & Full cohort & $\begin{array}{l}\text { Full cohort, } \\
\text { year } 2\end{array}$ & Completer cohort \\
\hline Patients $\mathrm{n}$ & 127 & 103 & 88 \\
\hline Age at enrolment years & $66.8 \pm 8.6$ & $66.7 \pm 8.7$ & $66.9 \pm 8.9$ \\
\hline Female sex & $59(46.5 \%)$ & $45(43.7 \%)$ & $37(42.0 \%)$ \\
\hline Smoking history pack-years & $47.0(33.7-60.0)$ & $50.4(34.0-60.0)$ & $50.0(33.5-58.5)$ \\
\hline Medication for COPD & $127(100 \%)$ & 103 (100\%) & $88(100 \%)$ \\
\hline Influenza vaccination during previous year & $114(89.8 \%)$ & $94(91.3 \%)$ & $82(93.2 \%)$ \\
\hline Pneumococcal vaccination during previous year & $12(9.4 \%)$ & $10(9.7 \%)$ & $8(9.1 \%)$ \\
\hline \multicolumn{4}{|l|}{ COPD status by GOLD stage } \\
\hline Mild & $0(0 \%)$ & $0(0 \%)$ & $0(0 \%)$ \\
\hline Moderate & $57(44.9 \%)$ & $46(44.7 \%)$ & $42(47.7 \%)$ \\
\hline Severe & $51(40.2 \%)$ & $44(42.7 \%)$ & $37(42.0 \%)$ \\
\hline Very severe & $19(15.0 \%)$ & $13(12.6 \%)$ & $9(10.2 \%)$ \\
\hline BODE index & $4(2-6)$ & $4(2-5)$ & $4(2-5)$ \\
\hline TLco predicted/actual $\mathrm{mmol} \cdot \mathrm{kPa}^{-1} \cdot \mathrm{min}^{-1}$ & $\begin{array}{c}7.9(7.2-8.8) / 4.5 \\
(3.4-5.8)\end{array}$ & $\begin{array}{c}7.9(7.4-9.0) / 4.5 \\
(3.5-6.1)\end{array}$ & $\begin{array}{c}8.0(7.4-8.9) / 4.5 \\
(3.6-6.0)\end{array}$ \\
\hline \multicolumn{4}{|l|}{$\begin{array}{l}\text { Subjects reporting exacerbations in } 12 \text { months } \\
\text { before study start }\end{array}$} \\
\hline One exacerbation & $28(22.0 \%)$ & $21(20.4 \%)$ & $20(22.7 \%)$ \\
\hline Two exacerbations & $37(29.1 \%)$ & $30(29.1 \%)$ & $28(31.8 \%)$ \\
\hline Three exacerbations & $25(19.7 \%)$ & $22(21.4 \%)$ & $17(19.3 \%)$ \\
\hline Four or more exacerbations & $37(29.1 \%)$ & $30(29.1 \%)$ & $23(26.1 \%)$ \\
\hline Exacerbations in preceding 12 months & $3.1 \pm 2.3 / 2(2-4)$ & $3.2 \pm 2.4 / 3(2-4)$ & $3.1 \pm 2.4 / 2(2-4)$ \\
\hline \multicolumn{4}{|l|}{$\begin{array}{l}\text { Exacerbations in preceding } 12 \text { months according } \\
\text { to severity }\end{array}$} \\
\hline Mild & $0.5 \pm 1.2$ & $0.4 \pm 1.2$ & $0.5 \pm 1.3$ \\
\hline Moderate & $2.3 \pm 1.9$ & $2.5 \pm 2.0$ & $2.3 \pm 2.0$ \\
\hline Severe & $0.4 \pm 0.6$ & $0.3 \pm 0.6$ & $0.3 \pm 0.5$ \\
\hline FEV 1 after bronchodilator use $\%$ predicted & $46.4 \pm 15.2$ & $46.7 \pm 14.6$ & $47.7 \pm 14.1$ \\
\hline
\end{tabular}

Data are presented as mean \pm SD, $\mathrm{n}(\%)$ and/or median (interquartile range), unless otherwise stated. COPD: chronic obstructive pulmonary disease; GOLD: Global Initiative for Chronic Obstructive Lung Disease; BODE: body mass index, obstruction, dyspnoea, exercise capacity; TLCO: transfer capacity of the lung for carbon monoxide; FEV1: forced expiratory volume in $1 \mathrm{~s}$.

relative to viral infection (figure 5 and table S3). For example, among frequent exacerbators, in sputum collected during exacerbation, bacterial infection was detected by culture in $60.0 \%$ (95\% CI $47.9-71.4 \%$ ) of patients in year 1 and $52.3 \%$ (95\% CI 41.9-62.5\%) in year 2, while viral infection was detected in $43.8 \%$ (95\% CI 32.0-56.0\%) in year 1 and $42.2 \%$ (95\% CI 31.9-52.9\%) in year 2. For infrequent exacerbators, bacterial infection was detected by culture in $76.5 \%$ (95\% CI 53.5-92.0\%) in year 1 and 50.0\% (95\% CI 26.9-73.1\%) in year 2, while viral infection was detected in $60.0 \%$ (95\% CI $35.1-87.1 \%$ ) in year 1 and $78.6 \%$ (95\% CI 53.4-94.2\%) in year 2. Evaluation of the presence of infection at stable visits relative to exacerbation frequency did not reveal any clear trends (figure 5 and table S3).

An absence of clear aetiology (bacterial or viral infection or eosinophils $>3 \%$ ) at exacerbation was detected more frequently in very frequent exacerbators (15.6\% (95\% CI 9.6-23.2\%) in year 1 and $16.1 \%(95 \%$ CI $6.1-31.5 \%)$ in year 2) than in frequent (6.3\% (95\% CI 2.0-13.9\%) and $12.0 \%$ (95\% CI 6.2-20.2), respectively) or infrequent exacerbators ( $0 \%$ in both years). Detection percentages were comparable among exacerbator groups at stable state (table S3).

\section{Influence of aetiological factors on change in yearly exacerbation frequency}

We examined the influence of bacterial or viral infection or eosinophilic inflammation at stable state on changes in exacerbation frequency from year 1 to year 2 in the completer cohort. Different rates of $H$. influenzae colonisation at stable state appeared to drive the likelihood of frequent exacerbation in year 2, a pattern that was not seen with $M$. catarrhalis colonisation, viral infection or eosinophilic inflammation (figure 6). For example, those with two or more additional exacerbations in year 2 versus year 1 were likely to have a higher rate of $H$. influenzae colonisation detected by culture $(7.2 \%$ increase in $H$. influenzae presence; OR 1.43, 95\% CI 0.71-2.91) than those with at least two fewer exacerbations in year 2 than in year 1 (8.5\% decrease; OR 0.63, 95\% CI 0.40-1.01) (difference between ORs, $\mathrm{p}=0.060$ ). For H. influenzae 


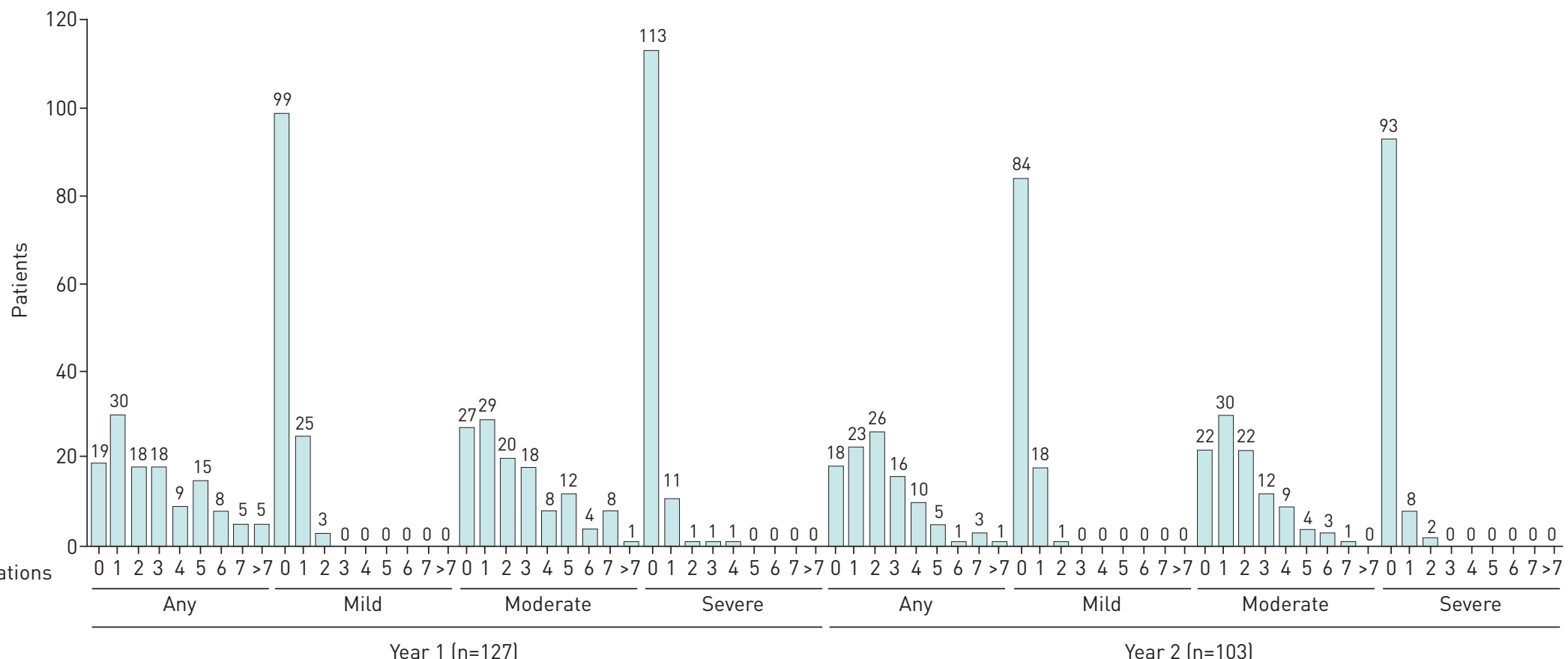

FIGURE 3 Distribution of patients in the first- or second-year follow-up according to the number of acute exacerbations of chronic obstructive pulmonary disease presented and their severity (full cohort, year 1 or year 2). The "any" category indicates overall classification; patients could experience exacerbations in one or more severity category. 


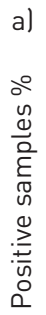
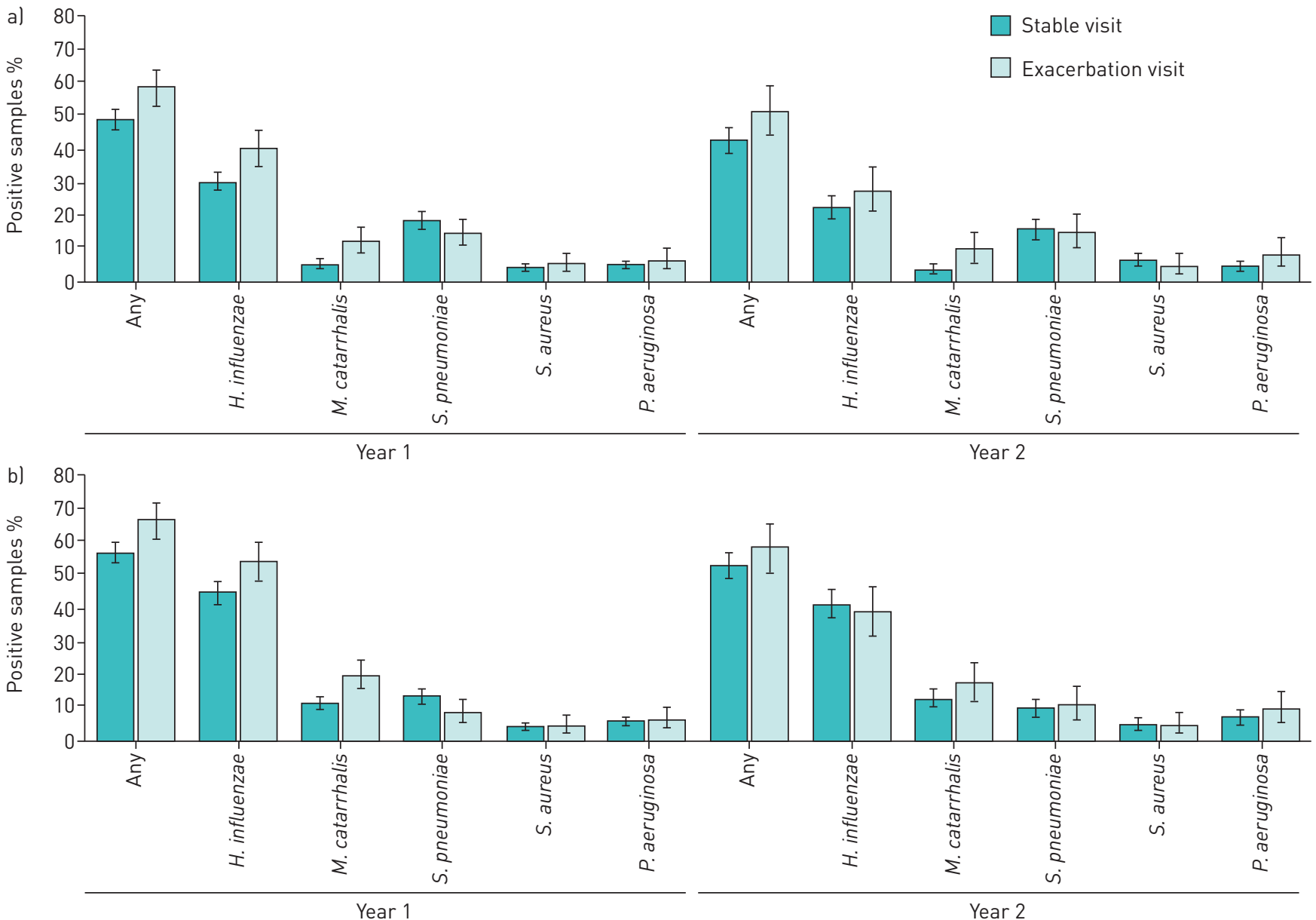

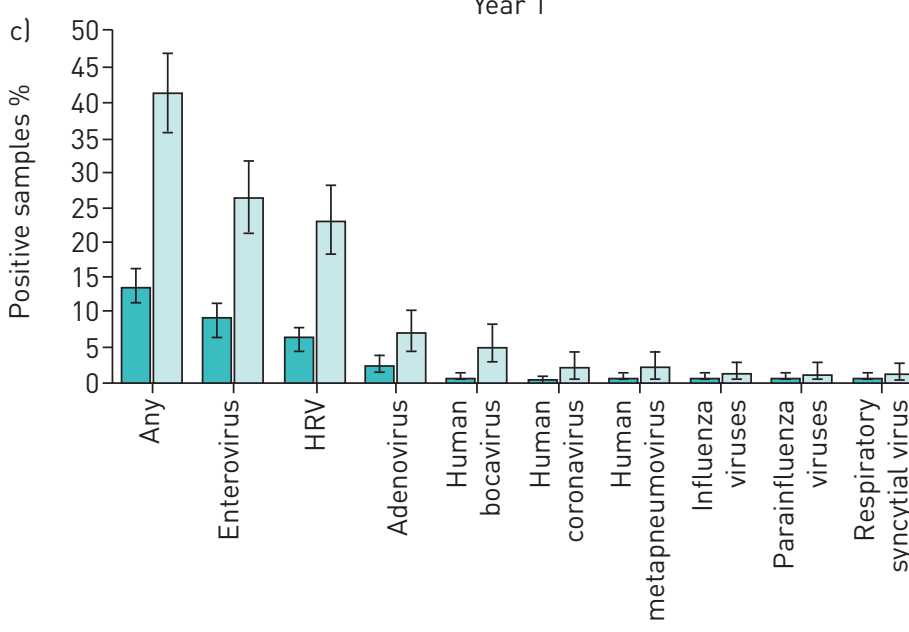

Year 1

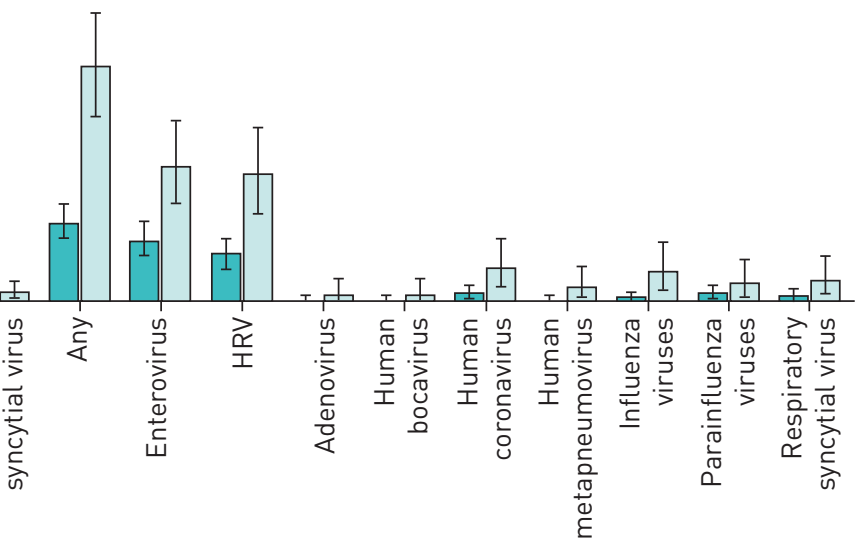

Year 2

FIGURE 4 Percentage of culture-positive or PCR-positive sputum samples at stable state and exacerbation in year 1 and year 2 (full cohort, year 1 or year 2). a) Percentage culture-positive for bacteria. b) Percentage PCR-positive for bacteria. c) Percentage PCR-positive for virus. Error bars represent $95 \%$ confidence intervals. HRV: human rhinovirus.

colonisation detected by PCR, there was a $13.1 \%$ increased detection (OR 1.76, 95\% CI $0.88-3.51$ ) for those with two or more additional exacerbations in year 2 versus year 1 and a $13.7 \%$ decrease (OR 0.56, 95\% CI 0.37-0.86) for those with at least two fewer exacerbations $(p=0.006)$. Repetition of this analysis for any study visit showed similar trends, with a slightly stronger effect for $H$. influenzae $(\mathrm{p}<0.01$ for both detection methods) (figure S1), possibly because of increased detection at exacerbation versus stable state (figure 4). 

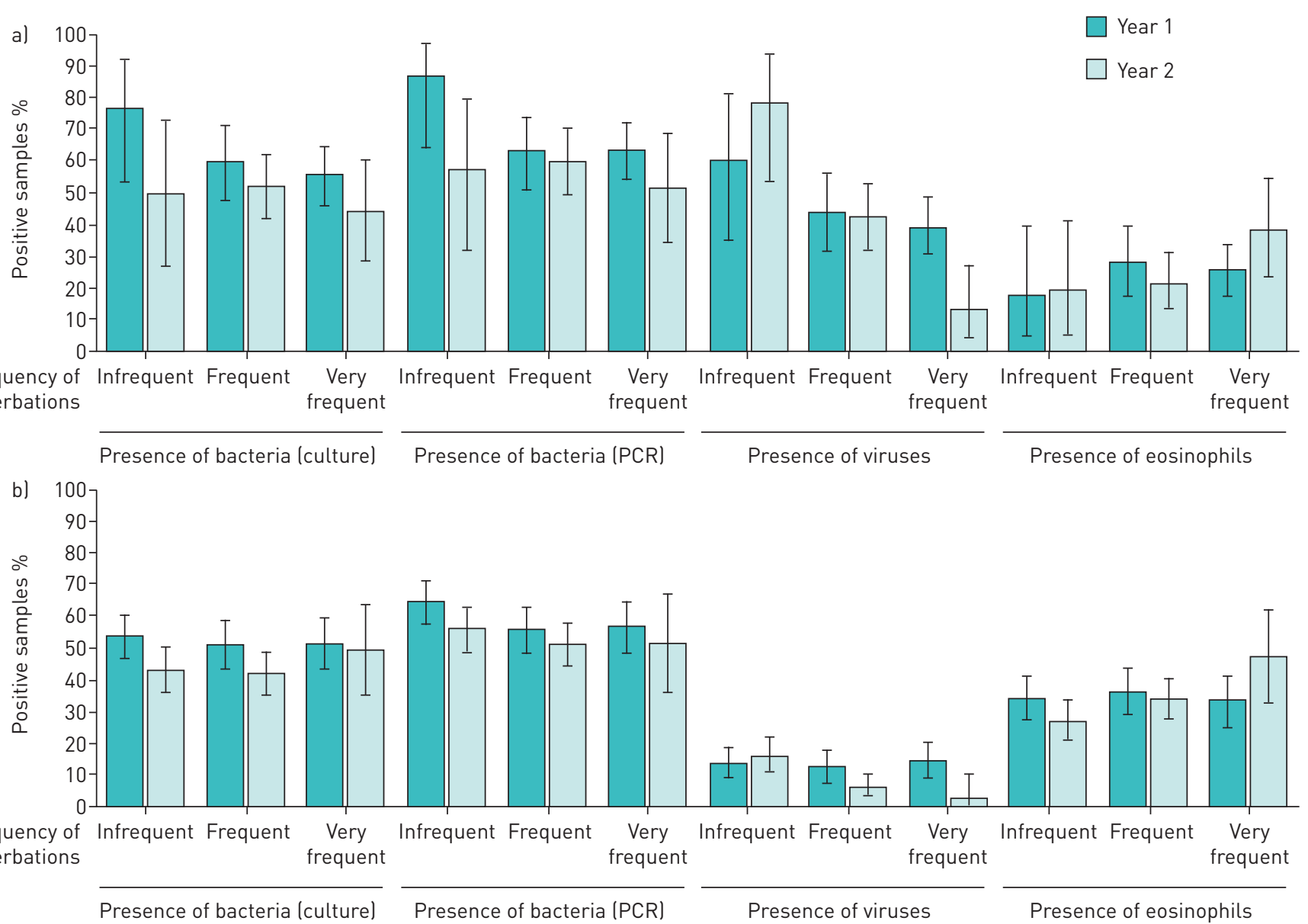

FIGURE 5 Percentage of chronic obstructive pulmonary disease patients with culture- or PCR-positive sputum samples for bacterial or viral pathogens or eosinophilic sputum samples (presence of eosinophils $>3 \%$ ) at a) exacerbation and b) stable visits, by frequency of exacerbations reported during year 1 or year 2 (completer cohort). Exacerbation frequency in specified year: infrequent, one or (for stable visits) none; frequent, two to four; very frequent, more than four. Error bars represent $95 \%$ confidence intervals.

Chronic macrolide use tended to be associated with fewer exacerbations in year 2. Among the 88 completers, 16 patients were administered chronic azithromycin treatment (six and 10 starting in year 1 and year 2, respectively). For those who started the antibiotic in year 1, the exacerbation rate decreased by at least two. In year 2, rates decreased or increased versus year 1, possibly because antibiotic treatments were initiated at different times, with variable patient follow-up. Ranking patients by difference in number of exacerbations in year 2 versus year 1 (table S4) showed those with at least two fewer exacerbations had the highest proportion of visits with chronic azithromycin treatment during the first (5.8\%) and second year $(18.6 \%)$. For those with one fewer exacerbation, the proportion of visits under azithromycin were, respectively, $0 \%$ and $15.6 \%$. No patients with the same or an increased exacerbation rate in year 2 received chronic azithromycin treatment in the first year.

\section{Discussion}

We used 2-year follow-up data from the AERIS study to evaluate, for the first time, how year-to-year variation in exacerbation frequency is influenced by the aetiology of AECOPD, thus gaining novel insights into the drivers of exacerbation frequency in an individual patient. Our analysis shows that infrequent exacerbators are likely to have high incidence of viral infection at exacerbation, while those with more frequent exacerbations tend to have a higher relative incidence of bacterially associated events. This suggests that bacterial infection at exacerbation may be a key driver of the frequent exacerbator phenotype rather than viruses and that, as exacerbation frequency increases, a higher proportion of events in an individual is likely to be bacterially related. This effect was not seen at stable state, which is possibly indicative of a relatively constant microbiome, as reported previously in clinically stable COPD patients $[10,15]$. In terms of viral infections, these are deemed to be stochastic events that occur following transmission and are identified less frequently than bacterial infection during an exacerbation [16]. 
a) H. influenzae PCR

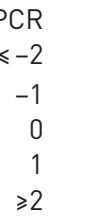

H. influenzae culture

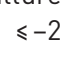

$\leqslant-2$

-1
0

0
1

$\geqslant 2$

$$
0.01
$$

$x^{2}+x^{2}$

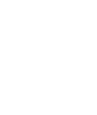

$\longmapsto$

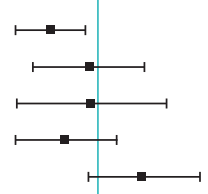

OR $(95 \% \mathrm{Cl})$

$0.564(0.368-0.859)$

0.895 (0.45-1.778)

0.93 (0.381-2.295)

$0.689(0.369-1.272)$

$1.758(0.884-3.508)$

$0.634(0.396-1.009)$

$0.235(0.1-0.553)$

$0.497(0.213-1.131)$

$0.607(0.286-1.251)$

$1.432(0.706-2.906)$

100

OR $(95 \% \mathrm{CI})$

b) M. catarrhalis PCR

$$
\begin{array}{r}
\leqslant-2 \\
-1 \\
0 \\
1 \\
\geqslant 2
\end{array}
$$

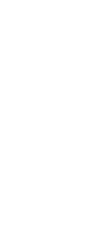

$\log$ OR

36 (1.635-5.906)

$1.032(0.315-3.285)$

0.239 (0.052-0.805)

0.595 (0.18-1.718)

$0.876(0.257-2.682)$

M. catarrhalis culture

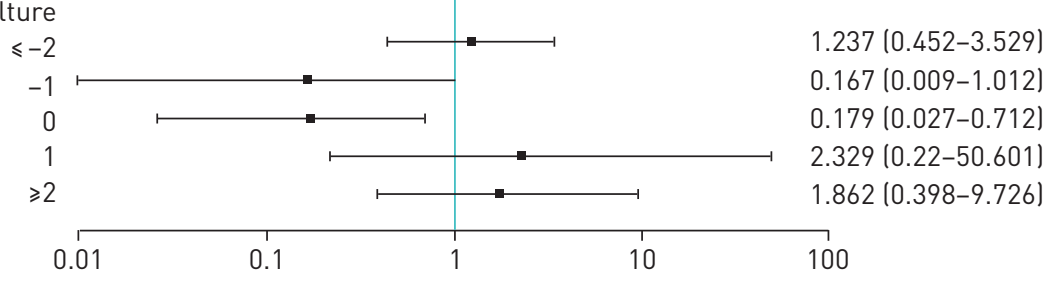

c)

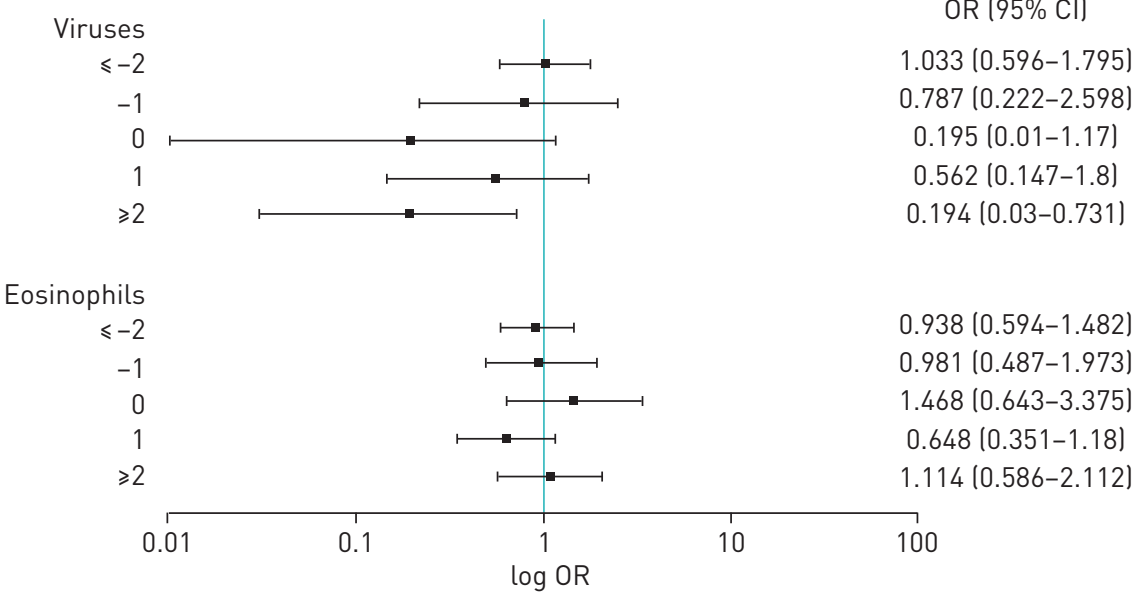

FIGURE 6 Effect of the presence of aetiological factors lbacterial or viral infection, or eosinophilic inflammation) at stable visits on the odds of experiencing a change in number of exacerbations in year 2 relative to year 1 (completer cohort). a) Haemophilus influenzae detected by PCR or culture. Comparison of $\leqslant-2$ and $\geqslant 2$ groups, $p=0.006$ (PCR) and $p=0.060$ (culture). b) Moraxella catarrhalis detected by PCR or culture. Comparison of $\leqslant-2$ and $\geqslant 2$ groups, $p=0.068$ (PCR) and 0.673 (culture). c) Viruses and eosinophils. Comparison of $\leqslant-2$ and $\geqslant 2$ groups, $p=0.052$ (viruses) and $p=0.669$ (eosinophils). $\leqslant-2$ : at least two fewer exacerbations in year 2 than in year $1 ;-1$ : one fewer exacerbation; 0 : no change; 1 : one more exacerbation; $\geqslant 2$ : at least two more exacerbations. Error bars represent $95 \%$ confidence intervals. p-values were estimated using the Wald test.

Bacterial and viral co-infection is associated with more impaired lung function and longer hospital stays than only bacterial or viral infection [17-19]. Therefore, viral infections alone are unlikely to drive the more frequent exacerbation phenotype. Among the very frequent exacerbation phenotype, exacerbations not related to infection or associated with eosinophilic inflammation were detected more often than in the 
frequent or infrequent exacerbation groups, possibly due to the frailty of these COPD patients and the impact of comorbidities in driving symptomatic deteriorations.

We also examined the effect of bacterial or viral infection or eosinophilic inflammation on a change in exacerbation frequency from one year to the next. H. influenzae colonisation at stable state appears to drive the likelihood of exacerbation since those with additional exacerbations in year 2 versus year 1 had a higher rate of $H$. influenzae colonisation at stable state than those who experienced no increase or fewer exacerbations in year 2 than in year 1. Consistent results were obtained for the full cohort, including patients with incomplete follow-up (data not shown). Persistent infection of the COPD airways with $H$. influenzae has been shown to contribute to chronic airway inflammation that increases symptoms and accelerates pulmonary disease progression [20]. Our results support chronic colonisation with $H$. influenzae as an important, potentially modifiable risk factor for exacerbations. The mechanism for this susceptibility to exacerbation in $H$. influenzae-colonised individuals may be partly related to an interaction with viral infection, as noted from previous AERIS study results [9], as well as independent effects. Moreover, the inflammatory milieu has been shown to be altered in the $H$. influenzae-colonised airway of COPD patients, with significantly greater levels of interleukin (IL)-1 $\beta$ in bronchoalveolar lavage samples than in COPD patients negative for $H$. influenzae [21]. This may be a direct result of infection and stimulation of inflammasomes and IL-1 $\beta$ secretion [22].

There were similarities year-to-year in relative incidence of the most common bacterial and viral pathogenic species in AECOPD. As expected, some variation in incidence of viruses was observed but HRV remained the most common virus detected in year 2. H. influenzae was the most common bacterial infection in both years, in line with previous study results [23-25]. However, the rate of exacerbations that were positive for $H$. influenzae was lower in the second year than in the first year for the full cohort and the same trend was observed for $M$. catarrhalis. This may be related to dissimilarities between the year 1 and year 2 populations, although no clear differences in cohort demographics were seen. The intensive nature of the study could have been a contributing factor since patients received a high level of clinical attention. Fewer patients with a very frequent exacerbator phenotype in year 2 suggests patients in the completer cohort may have been less frail in the second year than in year 1 . However, among patients with at least five exacerbations in the first year, only $21 \%$ did not complete two years of follow-up. Also, importantly, there is an indication of changes in clinical practice since stable state patients in the full cohort received azithromycin during 3.0\% of their follow up in year 1 compared with $11.5 \%$ in year 2 , which is likely to have altered airway microbial patterns and exacerbation frequency. Azithromycin use is clinically indicated by UK national guidelines [26] and our observation of a lower exacerbation rate in year 2 adds further support for the use of azithromycin in AECOPD treatment at least in the short term.

The strengths, including the early capture of exacerbations, and limitations of the AERIS study design were described in detail previously [9] and limitations specific to the year-to-year analyses have been discussed regarding possible clinical differences between the year 1 and year 2 study populations. With the stratification of patients according to exacerbator phenotype, the number of patients per exacerbation rate category was small. The results of these post hoc analyses should therefore be regarded as trends observed in the context of complex effects contributed by various aetiological factors, which need to be investigated further in larger prospective studies.

In conclusion, this year-to-year analysis of patients with COPD found that, whilst overall patterns of infection were similar across both years of the study, there were important differences within certain patients, which give valuable insights into the aetiology of exacerbations. By analysing the same cohort of patients over two years, we show for the first time that a change in yearly exacerbation rate may be associated with variations in $H$. influenzae colonisation. This confirms the importance of $H$. influenzae as a driver of exacerbation risk and identifies this bacterium as a modifiable factor and hence therapeutic target for future clinical trials of interventions to reduce the risk of exacerbation with this common, global disease.

Acknowledgements: The authors would like to thank all the study volunteers for their invaluable contribution towards furthering COPD knowledge and each team member for their assistance in conducting the study. We acknowledge all members of the AERIS study group. The authors thank the DDL Diagnostic Laboratory (Rijswijk, The Netherlands) and the GSK Clinical Laboratory Sciences teams for their contribution to the study assays. The authors thank the GSK teams that worked on the monitoring, follow-up and the logistics of the samples as well as data management. The authors would like to also thank Géraldine Drevon, Salomé Murinello and Regis Azizieh (XPE Pharma \& Science, Wavre, Belgium), on behalf of GSK (Rixensart, Belgium), for coordination and editorial support, and Joanne Knowles (independent writer, on behalf of GSK) for writing assistance.

The AERIS Study Group: J. Alnajar (University of Southampton, Southampton, UK), R. Anderson (University of Southampton, Southampton, UK), E. Aris (GSK, Wavre, Belgium), W.R. Ballou (GSK, Rockville, MD, USA), A. Barton (University Hospital Southampton NHS Foundation Trust, Southampton, UK), S. Bourne (University of Southampton, Southampton, and Portsmouth Hospitals NHS Trust, Portsmouth, UK), M. Caubet (GSK, Wavre, Belgium), S.C. Clarke 
(University of Southampton, Southampton, UK), D. Cleary (University of Southampton, Southampton, UK), C. Cohet (GSK, Wavre, Belgium), N.A. Coombs (University of Southampton, Southampton, UK), K. Osman (University of Southampton, Southampton, UK), J-M. Devaster (GSK, Wavre, Belgium), V. Devine (University of Southampton, Southampton, UK), N. Devos (GSK, Wavre, Belgium), E. Dineen (University Hospital Southampton NHS Foundation Trust, Southampton, UK), T. Elliott (University of Southampton, Southampton, UK), R. Gladstone (Wellcome Sanger Institute, Hinxton, UK), S. Harden (University Hospital Southampton NHS Foundation Trust, Southampton, UK), J. Jefferies (University of Southampton, Southampton, UK), V.L. Kim (University of Southampton and University Hospital Southampton NHS Foundation Trust, Southampton, UK), P. Moris (GSK, Wavre, Belgium), K.K. Ostridge (University of Southampton and University Hospital Southampton NHS Foundation Trust, Southampton, UK), T.G. Pascal (GSK, Wavre, Belgium), M. Peeters (GSK, Wavre, Belgium), B. Sente (GSK, Wavre, Belgium), S. Schoonbroodt (GSK, Wavre, Belgium), K.J. Staples (University of Southampton and University Hospital Southampton NHS Foundation Trust, Southampton, UK), A.C. Tuck (University of Southampton, Southampton, UK), L. Welch (Solent NHS trust, Southampton, UK), V. Weynants (GSK, Wavre, Belgium), T.M.A. Wilkinson (University of Southampton and University Hospital Southampton NHS Foundation Trust, Southampton, UK), A.P. Williams (University of Southampton, Southampton, UK), N.P. Williams (University of Southampton and University Hospital Southampton NHS Foundation Trust, Southampton, UK), C. Woelk (University of Southampton, Southampton, UK), M. Wojtas (University of Southampton, Southampton, UK) and S.A. Wootton (University Hospital Southampton NHS Foundation Trust, Southampton, UK). All members of the AERIS Study Group were involved in the planning, conduct and/or reporting of the work described in the article.

Author contributions: T.M.A. Wilkinson, E. Aris, S.C. Bourne, S.C. Clarke, T.G. Pascal, L. Taddei, A.C. Tuck, V.L. Kim, S.A. Wootton and J-M. Devaster were involved in the study conception and design. T.M.A. Wilkinson, E. Aris, S.C. Bourne, S.C. Clarke, M. Peeters, T.G. Pascal, A.C. Tuck, V.L. Kim, K.K. Ostridge, K.J. Staples, N.P. Williams, A.P. Williams, S.A. Wootton and J-M. Devaster were involved in acquisition and generation of data. T.M.A. Wilkinson, E. Aris, S.C. Bourne, S.C. Clarke, M. Peeters, T.G. Pascal, V.L. Kim, K.K. Ostridge, K.J. Staples, N.P. Williams, A.P. Williams, S.A. Wootton and J-M. Devaster were involved in data analysis and data interpretation. All authors contributed substantially to the development of the manuscript and approved the final version.

Conflict of interest: T.M.A. Wilkinson reports grants and nonfinancial support from GSK during the conduct of the study; and support for travel and meeting attendance from Boehringer Ingelheim, and grants and support for travel and meeting attendance from AstraZeneca, outside the submitted work. E. Aris is an employee of GSK. S.C. Bourne has nothing to disclose. S.C. Clarke reports grants from GSK, during the conduct of the study; and grants from Pfizer, outside the submitted work. M. Peeters is an employee of GSK. T.G. Pascal is an employee of GSK group of companies and owns restricted shares. L. Taddei has nothing to disclose. A.C. Tuck has nothing to disclose. V.L. Kim reports grants from GSK Biologicals SA during the conduct of the study and outside the submitted work. K.K. Ostridge has nothing to disclose. K.J. Staples reports grants from GSK Biologicals SA during the conduct of the study; and grants from Asthma UK (08/026), grants from BMA HC Roscoe Award, outside the submitted work. In addition, K.J. Staples has a patent PCT/GB2010/050821 "Ex Vivo Modelling of Therapeutic Interventions" pending. N.P. Williams has nothing to disclose. A.P. Williams has nothing to disclose. S.A. Wootton reports grants and nonfinancial support from GSK during the conduct of the study. J-M. Devaster is an employee of, and holds shares and restricted shares in GSK.

Support statement: The study funder, GlaxoSmithKline Biologicals SA, designed the study in collaboration with the investigators, and coordinated collection, analysis and interpretation of data. The investigators obtained data and cared for the study participants. The authors had full access to all data in the study, contributed to the writing of the report and had final responsibility for the decision to submit for publication. Funding information for this article has been deposited with the Crossref Funder Registry.

\section{References}

1 Mackay AJ, Hurst JR. COPD exacerbations: causes, prevention, and treatment. Immunol Allergy Clin North Am 2013; 33: 95-115.

2 Müllerova H, Maselli DJ, Locantore N, et al. Hospitalized exacerbations of COPD: risk factors and outcomes in the ECLIPSE cohort. Chest 2015; 147: 999-1007.

3 Global Initiative for Chronic Obstructive Lung Disease (GOLD). Global strategy for the diagnosis, management, and prevention of Chronic Obstructive pulmonary disease. 2017 report. http://goldcopd.org/download/326/. Date last updated: 2017. Date last accessed: December 9, 2018

4 Hurst JR, Vestbo J, Anzueto A, et al. Susceptibility to exacerbation in chronic obstructive pulmonary disease. N Engl J Med 2010; 363: 1128-1138.

5 Donaldson GC, Seemungal TA, Bhowmik A, et al. Relationship between exacerbation frequency and lung function decline in chronic obstructive pulmonary disease. Thorax 2002; 57: 847-852.

6 Wang $\mathrm{H}, \mathrm{Gu} \mathrm{X}$, Weng $\mathrm{Y}$, et al. Quantitative analysis of pathogens in the lower respiratory tract of patients with chronic obstructive pulmonary disease. BMC Pulm Med 2015; 15: 94.

7 Molyneaux PL, Mallia P, Cox MJ, et al. Outgrowth of the bacterial airway microbiome after rhinovirus exacerbation of chronic obstructive pulmonary disease. Am J Respir Crit Care Med 2013; 188: 1224-1231.

8 Millares L, Ferrari R, Gallego M, et al. Bronchial microbiome of severe COPD patients colonised by Pseudomonas aeruginosa. Eur J Clin Microbiol Infect Dis 2014; 33: 1101-1111.

9 Wilkinson TMA, Aris E, Bourne S, et al. A prospective, observational cohort study of the seasonal dynamics of airway pathogens in the aetiology of exacerbations in COPD. Thorax 2017; 72: 919-927.

10 Mayhew D, Devos N, Lambert C, et al. Longitudinal profiling of the lung microbiome in the AERIS study demonstrates repeatability of bacterial and eosinophilic COPD exacerbations. Thorax 2018; 73: 422-430.

11 Bourne S, Cohet C, Kim V, et al. Acute Exacerbation and Respiratory InfectionS in COPD (AERIS): protocol for a prospective, observational cohort study. BMJ Open 2014; 4: e004546.

12 Kim VL, Coombs NA, Staples KJ, et al. Impact and associations of eosinophilic inflammation in COPD: analysis of the AERIS cohort. Eur Respir J 2017; 50: 1700853. 
13 Vestbo J, Hurd SS, Agusti AG, et al. Global strategy for the diagnosis, management, and prevention of chronic obstructive pulmonary disease: GOLD executive summary. Am J Respir Crit Care Med 2013; 187: 347-365.

14 Bafadhel M, McKenna S, Terry S, et al. Acute exacerbations of chronic obstructive pulmonary disease: identification of biologic clusters and their biomarkers. Am J Respir Crit Care Med 2011; 184: 662-671.

15 Sinha R, Weissenburger-Moser LA, Clarke JL, et al. Short term dynamics of the sputum microbiome among COPD patients. PLoS One 2018; 13: e0191499.

16 Segal LN, Martinez FJ. Chronic obstructive pulmonary disease subpopulations and phenotyping. J Allergy Clin Immunol 2018; 141: 1961-1971.

17 Hewitt R, Farne H, Ritchie A, et al. The role of viral infections in exacerbations of chronic obstructive pulmonary disease and asthma. Ther Adv Respir Dis 2016; 10: 158-174.

18 Papi A, Bellettato CM, Braccioni F, et al. Infections and airway inflammation in chronic obstructive pulmonary disease severe exacerbations. Am J Respir Crit Care Med 2006; 173: 1114-1121.

19 Wilkinson TM, Hurst JR, Perera WR, et al. Effect of interactions between lower airway bacterial and rhinoviral infection in exacerbations of COPD. Chest 2006; 129: 317-324.

20 Ahearn CP, Gallo MC, Murphy TF. Insights on persistent airway infection by non-typeable Haemophilus influenzae in chronic obstructive pulmonary disease. Pathog Dis 2017; 75.

21 Staples KJ, Taylor S, Thomas S, et al. Relationships between mucosal antibodies, non-typeable Haemophilus influenzae (NTHi) infection and airway inflammation in COPD. PLoS One 2016; 11: e0167250.

22 Rotta Detto Loria J, Rohmann K, Droemann D, et al. Nontypeable Haemophilus influenzae infection upregulates the NLRP3 inflammasome and leads to caspase-1-dependent secretion of interleukin-1 $\beta$ - A possible pathway of exacerbations in COPD. PLoS ONE 2013; 8: e66818.

23 Finney LJ, Ritchie A, Pollard E, et al. Lower airway colonization and inflammatory response in COPD: a focus on Haemophilus influenzae. Int J Chron Obstruct Pulmon Dis 2014; 9: 1119-1132.

24 Bafadhel M, Haldar K, Barker B, et al. Airway bacteria measured by quantitative polymerase chain reaction and culture in patients with stable COPD: relationship with neutrophilic airway inflammation, exacerbation frequency, and lung function. Int J Chron Obstruct Pulmon Dis 2015; 10: 1075-1083.

25 Barker BL, Haldar K, Patel H, et al. Association between pathogens detected using quantitative polymerase chain reaction with airway inflammation in COPD at stable state and exacerbations. Chest 2015; 147: 46-55.

26 National Institute for Health and Care Excellence. Chronic obstructive pulmonary disease in over 16s: diagnosis and management. Clinical guideline. www.nice.org.uk/guidance/NG115. Date last updated: December 2018. Date last accessed: December 92018 